\title{
Face Recognition based on Oriented Complex Wavelets and FFT
}

\author{
Rangaswamy $Y$ \\ Alpha College of Engineering \\ VTU University \\ Bangalore-077
}

\author{
Raja K B \\ UVCE, Bangalore. \\ Bangalore University \\ Bangalore-01
}

\author{
Venugopal K R \\ UVCE, Bangalore. \\ Bangalore University \\ Bangalore-01
}

\begin{abstract}
The Face is a physiological Biometric trait used in Biometric System. In this paper face recognition using oriented complex wavelets and Fast Fourier Transform (FROCF) is proposed. The five-level Dual Tree Complex Wavelet Transform(DTCWT) is applied on face images to get shift invariant and directional features along $\pm 15^{\circ}, \pm 45^{\circ}$ and $\pm 75^{\circ}$ angular directions. The different pose, illumination and expression variations of face images are represented in frequency domain using Fast Fourier Transform(FFT) resulting in FFT features. Features of DTCWT and FFT are fused by arithmetic addition to get final features. Euclidean Distance classifier is applied to the features to recognize the genuine and imposter faces. The Performance analysis of proposed method is tested with ORL, JAFFE, L-SPACEK and CMU-PIE having different illumination and pose conditions. The Results shows that Recognition Rate of proposed FROCF is better compared to Existing Recognition Methods.
\end{abstract}

\section{General Terms}

Euclidean Distance, Identification, False Acceptance Rate (FAR), False Rejection Rate (FRR), Total Success Rate(TSR),Recognition Rate, Equal Error Rate (EER).

\section{Keywords}

Biometrics, Face Recognition, DTCWT, FFT, TSR

\section{INTRODUCTION}

Biometrics is a secure and reliable authentication system based on Physiological and behavioral characterstics of an individual. Biometrics acquires a prominent accessing technique in modern security applications like access to banks, ATM, computers and other transactions which needs authorized user for a claimed service.The advantage of biometric based access to data over a traditional identification methods of ID cards and passwords is more secure as it cannot be shared and attacked by other than claimed authority. Biometrics works mainly on two biological characterstics namely physiological characterstics which include face, fingerprint and iris. The features extracted from these biological traits remains constant over a period of time compared to behavioral characterstics like gait, keystroke and voice which are liable to change based on circumstance and Enviornamental conditions. The selection of a good biometric trait depends on its distinctness, Easy availability and universal acceptance.

Face is widely used biometric trait for personal authentication because of its easy collectability and can be captured from longer distance. The face recognition has to face a challenges of recognizing an individual by extracting features which are robust to pose, illumination and expression variations. The features extracted are subjected to Region of Operating Characterstics which gives performance parameters False Acceptance Rate (FAR), False Rejection Rate (FRR) and True Success Rate. The efficient biometric system with minimum FAR and FRR and maximum TSR can be achieved by selecting a good classifier for a matching.In the proposed Face Recognition based on Oriented Complex Wavelets and FFT method DTCWT and FFT features are fused to get better Recognition Rates compared to existing state of the art methods.

\section{RELATED WORK}

Alaa Eleyan et al., [1] have proposed face recognition based on the Dual-Tree Complex Wavelet Transform (DT-CWT) to extract features from face images. Gabor wavelet features are extracted with DT-CWT using Gabor wavelets and similar kernels. The final features extracted from PCA components applied on DT-CWT coefficients gives better recognition rates. Zhongxi Sun et al., [2] have proposed face recognition using DTCWT features with 2DPCA. DTCWT has advantage over DWT as it provides local multi-scale description of images with good directional selectivity and shift invariance. DTCWT is robust to illumination variations and facial expression changes. Results have shown that using this technique it's possible to capture the local information of different position, orientation and scales.Ravi et al., [3] proposed the method in which the original face image is preprocessed and five level DT-CWT is applied in ordered to get DT-CWT coefficients. The $3 \times 3$ matrix is considered, for each of these matrix local binary patterns is applied to get the final features and comparison of features of test image with database image is done using Euclidean Distance.

Yuehui Sun and Minghui Du[4] have introduced a face recognition based on DT-CWT feature representation using Orthogonal Neighborhood Preserving Projections (ONPP), ONPP is a linear dimensionality reduction technique that attempts to intrinsic Neighborhood geometry of data samples and the global geometry. Sun Zhongxi et al., [5] have introduced a face recognition by combining DT-CWT and two dimensional inverse Fisher Discriminant Analysis. DTCWT is applied to extract features at different scales and orientations. 2DIFDA is applied for dimensionality reduction and feature selection. Raja and Ramesha [6] have presented performance evaluation of face recognition based on DTCWT using multi-matching classifiers. Nick Kingsbury [7] has discussed a method to design a filters for DT-CWT. The filters are designed such that both the filers are of even length and also time-reverse of each other. The filters have additional property that the group delay between the filter is one quarter of the sample period.Uzair et al.,[8] proposed a hyper spectral face recognition algorithm based on spatio spectral 
covariance for band fusion and PLS regression for classification. The algorithm was tested on three standard databases and compared with 18 existing state of the art algorithms including seven image set classification, six gray scale/RGB and five hyper spectral face recognition algorithms. Gao Zhirong et al., [9] proposed a multiple feature fusion and linear regression based face recognition based on transform domain features which provides a comprehensive face information and decrease the effect of pose and illumination variations. The linear regression classifier is used for recognition. Yue Long et al., [10] proposed a recognition method using sparse representation on down sampled input image to locate un occluded face parts. Local stastical features are obtained by applying Linear Discriminant Analysis on occluded face parts for better recognition rate. Jun Huang et al., [11] proposed a face recognition algorithm based on both the Multilinear Principal Component Analysis (MPCA) and Linear Discriminant Analysis(LDA). This approach treats face images as multidimensional tensor in order to find the optimal tensor subspace for dimension reduction. The LDA is used to project samples to new discriminant feature space and $\mathrm{K}$ Nearest Neighbor (KNN) classifier is used for matching. Zhang et al., [12] proposed a face recognition based on singular value decomposition features which reduces and remove redundancy in a data using a nearest orthogonal matrix representation. The nearest neighbor classifier is used for matching

\section{BACKGROUND}

\subsection{Dual Tree Complex Wavelet Transform}

Dual Tree Complex Wavelet Transform is a analytic transform with complex valued oscillating sinusoid basis functions producing oriented and complex coefficients which are robust to oscillations and shift variance.These Oriented complex wavelets effectively captures more directional features compared to 2D separable real wavelet transform. The filters selected to design oriented and complex wavelets should be (i) orthogonal (ii) approximately half sample delay between filter pairs (iii) finite support (iv) vanishing moments with linear phase for a perfect reconstruction of an image with limited redundancy.

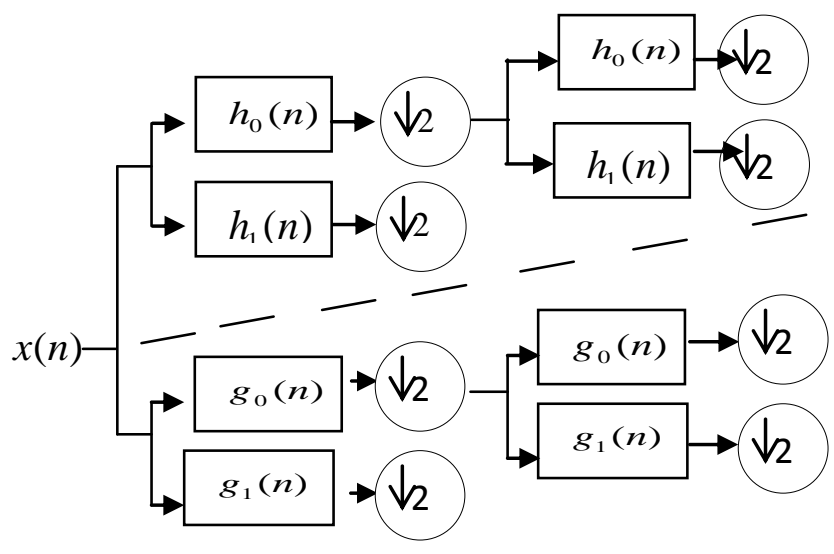

Imaginary Tree

Fig 1: Filter Bank structure for 2-Level DTCWT

DTCWT can be implemented using 2D separable two real wavelet transform in parallel as shown in Figure 1. The first real wavelet transform can be implemented using low pass and high pass filter coefficients $h_{0}(n)$ and $h_{1}(n)$ applied along row and column dimension of 2D data which forms Upper Filter bank structure of DTCWT. The second Real wavelet transform which represents Lower Filter bank of DTCWT can be implemented using low pass and high pass filter coefficients $g_{0}(n)$ and $g_{1}(n)$ which are approximately analytic to upper filter bank coefficients resulting in perfect reconstruction of input image data.

Real Oriented 2-D Dual-Tree Wavelet Transform: The Real 2-D Dual-Tree Wavelet Transform based on six oriented wavelets can be implemented using two real separable 2-D wavelet transform in parallel. To obtain a real 2-D wavelets oriented at $\pm 15, \pm 45, \pm 75$ a 2 -D separable wavelet transform is applied on image along row and column dimensions using six complex oriented wavelets as given in Equations (1) and (2).

$$
\begin{gathered}
\psi_{i}(x, y)=\frac{1}{\sqrt{2}}\left(\psi_{1, i}(x, y)-\psi_{2, i}(x, y)\right) \ldots \\
\psi_{i+3}(x, y)=\frac{1}{\sqrt{2}}\left(\psi_{1, i}(x, y)+\psi_{2, i}(x, y)\right) \ldots
\end{gathered}
$$

For $\mathrm{i}=1,2,3$, where the two separable 2-D wavelet are defined as given in Equations (3), (4) and (5).

$$
\begin{gathered}
\psi_{1,1}(x, y)=\phi_{h}(x) \psi_{h}(y), \psi_{2,1}(x, y)=\phi_{g}(x) \psi_{g}(y) . \\
\psi_{1,2}(x, y)=\psi_{h}(x) \phi_{h}(y), \quad \psi_{2,2}(x, y)=\psi_{g}(x) \phi_{g}(y) \\
\psi_{1,3}(x, y)=\phi_{h}(x) \psi_{h}(y), \psi_{2,3}(x, y)=\psi_{g}(x) \psi_{g}(y)
\end{gathered}
$$

Complex Oriented 2-D Dual-Tree Wavelet Transform: The complex 2-D dual-tree DWT is obtained by converting a 2D real wavelet transform into a complex wavelet transform of unidirectional spectrum. The real and imaginary part of this complex wavelet contains sum of two separable wavelets, the spectrum of theses separable wavelets are unidirectional avoiding checkerboard artifacts in frequency plane. The six angular directions $\pm 15^{\circ}, \pm 45^{\circ}$ and $\pm 75^{\circ}$ can be obtained by considering real and imaginary part of this complex wavelets. The Equations (6)-(11) gives six complex wavelets for these six directions where $h$ and $g$ indicates upper and lower tree filter coefficients respectively.

$$
\begin{aligned}
& \psi_{1}(x, y)=\left[\psi_{h}(x) \psi_{h}(y)-\psi_{g}(x) \psi_{g}(y)\right]+j\left[\psi_{h}(x) \psi_{g}(y)+\psi_{g}(x) \psi_{h}(y)\right] \ldots \ldots . . .(6) \\
& \psi_{2}(x, y)=\left[\psi_{h}(x) \psi_{h}(y)+\psi_{g}(x) \psi_{g}(y)\right]+j\left[\psi_{h}(x) \psi_{g}(y)-\psi_{g}(x) \psi_{h}(y)\right] \ldots \ldots . . \quad(7) \\
& \psi_{3}(x, y)=\left[\phi_{h}(x) \psi_{h}(y)-\phi_{g}(x) \psi_{g}(y)\right]+j\left[\phi_{g}(x) \psi_{g}(y)+\phi_{h}(x) \psi_{h}(y)\right] \ldots \ldots \quad(8) \\
& \psi_{4}(x, y)=\left[\phi_{h}(x) \psi_{h}(y)+\phi_{g}(x) \psi_{g}(y)\right]+j\left[\phi_{g}(x) \psi_{g}(y)-\phi_{h}(x) \psi_{h}(y)\right] \ldots .(9) \\
& \psi_{5}(x, y)=\left[\psi_{h}(x) \phi_{h}(y)-\psi_{g}(x) \phi_{g}(y)\right]+j\left[\psi_{g}(x) \phi_{g}(y)+\psi_{h}(x) \phi_{h}(y)\right] \ldots . .(10) \\
& \psi_{6}(x, y)=\left[\psi_{h}(x) \phi_{h}(y)+\psi_{g}(x) \phi_{g}(y)\right]+j\left[\psi_{g}(x) \phi_{g}(y)-\psi_{h}(x) \phi_{h}(y)\right] \ldots(11)
\end{aligned}
$$




\subsection{Fast Fourier Transform}

Fast Fourier Transform is a fast computation algorithm for Discrete Fourier Transform (DFT). DFT is used in signal processing applications like Linear Filtering, Correlation analysis and spectrum analysis that involves more computation time resulting in less efficient algorithms. In FFT, data sequence is decomposed into small sequences till we get single-point sequences. For $N=2^{v}$, this decomposition can be performed $v=\log _{2} N$ times. Thus, the total number of complex multiplications is reduced to $(N / 2) \log _{2} N$ against $\mathrm{N}^{2}$ complex multiplication of direct computation of DFT. Similarly, the number of complex additions is reduced to $N \log _{2} N$ compared to $\mathrm{N}^{2}-\mathrm{N}$ complex additions of direct DFT computation. In the proposed FROCF algorithm the two dimensional FFT is applied on 128*512 face images and the resulting complex coefficient matrix is converted into one dimentional vector of size 65,530 which forms FFT features.

\section{PROPOSED MODEL}

In this section, the proposed FROCF model is discussed. The transform domain features are fused to generate final feature set in the Proposed Model to enhance the recognition rate. The block diagram of proposed FROCF is shown in Figure 2.

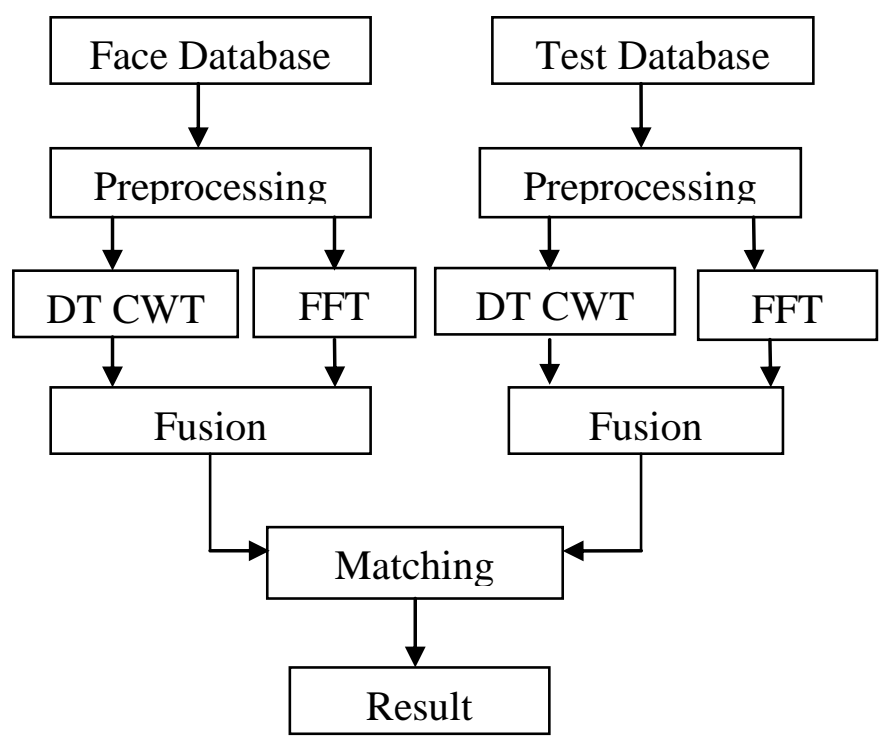

Fig 2: The Block Diagram of Proposed FROCF Model

\subsection{Face database}

The face database such as ORL, JAFFE, L-Speck and CPUPIE are used for the Performance analysis.

\subsubsection{ORL database}

The database has 40 Persons with ten images per Person having size of $112 \times 92$. The database is created by considering 20 persons with 9 images per person ie 180 face images in the database. The tenth image of every person is considered as test image. The samples of ORL database of person is shown in the Figure 3.

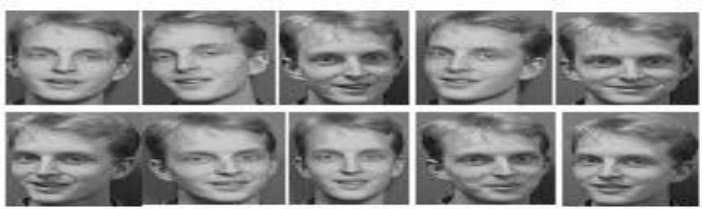

Fig 3 : Samples of ORL Database

\subsubsection{JAFFE database}

The JAFEE database has ten person with twenty images per person having each image size of 256x256. The database is created by considering five persons with 19 images per person i.e, total no of images in the database are 95. The sample of JAFFE database of a person is as shown in the Figure 4.

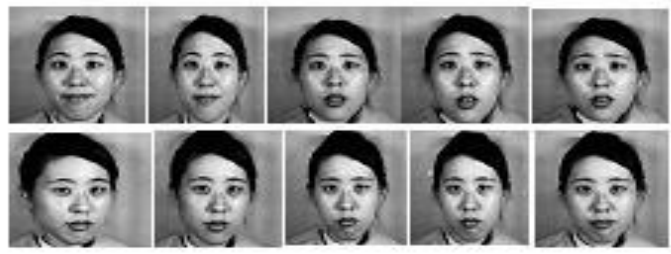

Fig 4: Samples of JAFFE database

\subsubsection{Lspeck-database}

The database has 120 persons with twenty image samples per person. The database is created by considering 20 persons with 19 images ie., total no of samples in the database are 380.The image samples of L-spacek database of a person is as shown in Figure 5.
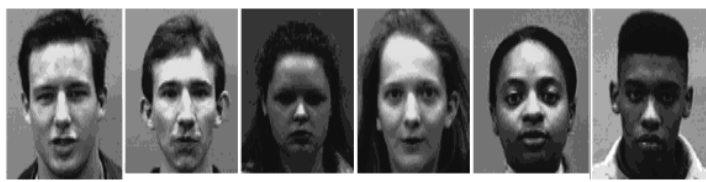

Fig 5: Samples of $L$-space $K$ database

\subsubsection{CMU-PIE database}

The database has 68 persons with 13 images per person having each image size of $640 \times 480$. The database is created by considering 15 persons with eight images per person i.e, total no images in the database are 120.The samples of CMUPIE database of a person is as shown in Figure 6.

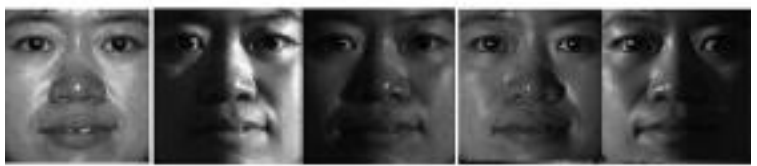

Fig 6 : Samples of CMU-PIE Database

\subsection{Preprocessing}

The different face databases has different dimensions in the face, hence the images are resized into uniform size. The each image is resized to $2^{m} \times 2^{n}$ to apply DT-CWT. Where $\mathrm{m}$ and $\mathrm{n}$ are integers. The face images are resized to $2^{7} \times 2^{9}$ ie $128 \times 512$.

\subsection{Feature Extraction by DTCWT}

The Feature Extraction of 2D DTCWT consists of following three steps.

(i) An input image is decomposed using 2D DWT. In the proposed model 5 level DT-CWT is applied on face image, which provides sixteen sub bands at each level, 4 sub bands of low frequencies and 12 sub bands of high frequencies. At every level, the image size is reduced to half of the original image size. i.e., after 5 levels the image size is reduced to $4 \times 16$.

(ii) In the second step, every two corresponding sub bands which have the same pass bands are linearly combined by averaging and differencing. As a result, the sub bands of 2D-CWT at each level are obtained as $\left(\mathrm{LH}_{\mathrm{a}}+\mathrm{LH}_{\mathrm{b}}\right) / \sqrt{2}$, 


$$
\begin{aligned}
& \left(\mathrm{LH}_{\mathrm{a}}-\mathrm{LH}_{\mathrm{b}}\right) / \sqrt{2}, \\
& \left(\mathrm{HH}_{\mathrm{a}}+\mathrm{HH}_{\mathrm{b}}\right) / \sqrt{2}
\end{aligned}
$$

(iii) Magnitudes of real and imaginary bands are used as features for face recognition.

The 2D separable DWT on each decomposition produces three high frequency bands HL,HL and $\mathrm{HH}$ as shown in Figure 9 which gives a directional information along $0^{\circ}, \pm 45^{\circ}$, and $90^{\circ}$. DTCWT implemented using 2D real wavelet transform produces six complex wavelets producing directional information along $\pm 15^{\circ}, \pm 45^{\circ}$ and $\pm 75^{\circ}$ directions by taking real and imaginary part of each of these complex wavelets. The magnitude of real and imaginary part of a set of six complex wavelets are calculated using Equation (12) and Equation (13) Final magnitude coefficients are obtained by concatenating using Equation (14).

$$
\begin{aligned}
& m_{a c}=\sqrt{m_{a}{ }^{2}+m_{c}{ }^{2}} \ldots \ldots \ldots \ldots \ldots . \\
& m_{b d}=\sqrt{m_{b}{ }^{2}+m_{d}{ }^{2}} \ldots \ldots \ldots \ldots \ldots \\
& M=\left[m_{a c} ; m_{b d}\right] \ldots \ldots \ldots \ldots \ldots .
\end{aligned}
$$

Where $m_{a}, m_{b}$ and $m_{c}, m_{d}$ are corresponds DTCWT high frequency coefficient vectors of size $1 \times 192$ at 5-Level DTCWT. The Final feature vector $M$ of size $1 \times 384$ is obtained by concatenating magnitude of $m_{a c}$ and $m_{b d}$.

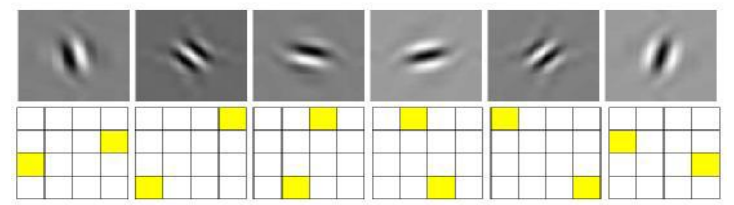

Fig 9: Showing Orientation of complex wavelets

\subsection{FFT Feature Extraction}

The FFT is applied on preprocessed face image of size $128 \times 512$ to generate FFT coefficients. The complex FFT coefficients are converted into absolute values and FFT matrix is converted into 1-dimenstional column vector of size $65,536 \times 1$. The absolute fft coefficient values are sorted in the descending order. The top dominant 384 coefficients are considered as FFT features.

\subsection{Final Futures.}

The DT-CWT features are fused with dominant FFT features using arithmetic addition to generate final features, for better recognition of a person as given by Equation (15)

$$
\text { Final Feature }=\sum_{i=1}^{384}\left(D T C W T_{i}+F F T_{i}\right) \ldots
$$

\subsection{Matching}

The test images features obtained using 5-Level DTCWT and FFT are compared with features of database images using Euclidian distance to compute FRR, FAR, EER and TSR values. The Euclidian distance is given in the Equation (16)

$$
d(p, q)=\sqrt{\sum_{i}^{n}\left(p_{i}-q_{i}\right)^{2}}
$$

Where

$$
\begin{aligned}
& \mathrm{p}_{\mathrm{i}}=\text { the feature value of the database images. } \\
& \mathrm{q}_{\mathrm{i}}=\text { the feature value of the test image. }
\end{aligned}
$$

\section{ALGORITHM}

In this section the problem definition, objectives and algorithm are described. The Proposed algorithm for face recognition is given in Table 1

Problem Definition: Given face images to verify the authentication of a person using fusion of DTCWT and dominant FFT features

The objectives are:

(i) To increase the Total Success Rate (TSR).

(i) To reduce the False Rejection Rate (FRR).

(ii) To reduce the False Acceptance Rate (FAR)

Table 1 .Algorithm for proposed FROCF model

Input: Face images of ORL, L-Space k, JAFFE, CMP-PIE

Output: Face image identification

Step 1: The Face database is created.

Step 2:The face image is resized to $128 \times 512$

Step 3: The 5-Level DTCWT is applied to extract DT-CWT 384 coefficients.

Step 4: The FFT is applied on preprocessed image to generate absolute coefficient values and converted into 1dimestional column vector of size 65,536x1.

Step 5: The absolute coefficients are sorted in descending order and top 384 coefficients are considered as FFT features.

Step 6: The FFT and DT-CWT features are fused using arithmetic addition to generate final set of features.

Step 7: The features of test image are compared with features of database images using Euclidian distance to compare performance parameters.

\section{PER FORMANCE ANALYSIS}

In this section, the performance analysis of proposed algorithm is discussed. The face databases ORL, L-space K, and JAFFE and CMU-PIE are considered for performance analysis.

\subsection{Results for ORL Database}

FRR, FAR and TSR variations with threshold for ORL database are given in Table 2 . where out of 40 persons 20 are considered inside the database and 20 persons out of database for testing.. FRR and TSR decreases with increase in threshold whereas FAR increases with increase in threshold. As threshold increases, TSR increases from $0 \%$ to $95 \%$. The maximum TSR obtained for ORL database is $95 \%$.Figure 9 shows variations of False Rejection Rate and False 
Acceptance Rate with threshold. For the threshold value of 5 the FRR is $0 \%$ and FAR is $100 \%$. The TSR for ORL database is obtained at a threshold value of 2.25 is $90 \%$. Equal Error Rate obtained at this threshold value is $5 \%$.

Table 2. FRR, TSR and FAR variations with threshold for ORL database

\begin{tabular}{|c|c|c|c|}
\hline Threshold & FRR & TSR & FAR \\
\hline 0 & 1 & 0 & 0 \\
\hline 0.25 & 1 & 0 & 0 \\
\hline 0.50 & 1 & 0 & 0 \\
\hline 0.75 & 1 & 0 & 0 \\
\hline 1 & 0.8 & 0.2 & 0 \\
\hline 1.25 & 0.7 & 0.3 & 0 \\
\hline 1.50 & 0.5 & 0.5 & 0 \\
\hline 1.75 & 0.4 & 0.6 & 0 \\
\hline 2 & 0.1 & 0.9 & 0 \\
\hline 2.25 & 0.05 & 0.9 & 0 \\
\hline 2.50 & 0.05 & 0.9 & 0.65 \\
\hline 2.75 & 0.05 & 0.95 & 0.80 \\
\hline 3 & 0 & 0.95 & 0.85 \\
\hline 3.25 & 0 & 0.95 & 0.95 \\
\hline 3.50 & 0 & 0.95 & 0.95 \\
\hline 3.75 & 0 & 0.95 & 1 \\
\hline 4 & 0 & 0.95 & 1 \\
\hline
\end{tabular}

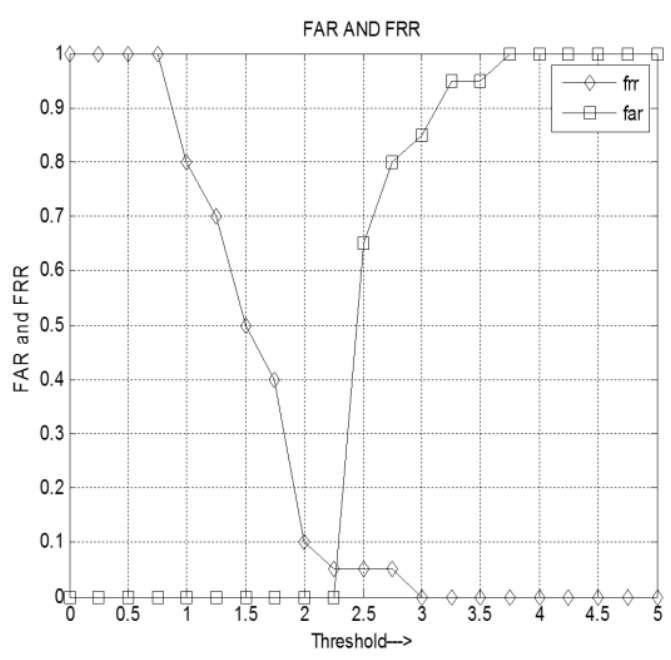

Fig 9: Graph of FRR and FAR with threshold value for ORL database

\subsection{Results for L-space $k$ Database}

The Table 3 shows the variations of FRR, TSR and FAR with threshold for L-spacek database where out of 80 persons 40 are considered inside the database and 40 persons out of database for testing. FRR and TSR decreases with increase in threshold whereas FAR increases with increase in threshold. As threshold increases, TSR increases from $0 \%$ to $97.5 \%$. The maximum TSR for $L$-space $K$ database is $97.5 \%$.The Figure 10 shows the variations of False Rejection Rate and False Acceptance Rate with threshold. For the threshold value of 5 the FRR is $0 \%$ and FAR is $100 \%$. The TSR for L-space K database is obtained at a threshold value of 2.1 is $95 \%$. EER obtained at this threshold value is $4 \%$.The Recognition Rate for L-speck database in terms of TSR values increases linearly from $20 \%$ to $97.5 \%$ as the pose, illumination variations and facial expressions of face images in a database are very less compared to variations of face images of ORL databases. The
TSR value reaches its maximum saturation value of $100 \%$ from a threshold value of 4 to 5 .

Table 3. FRR, TSR and FAR variations with Threshold for L-Spacek database

\begin{tabular}{|c|c|c|c|}
\hline Threshold & FRR & TSR & FAR \\
\hline 0 & 1 & 0 & 0 \\
\hline 0.25 & 1 & 0 & 0 \\
\hline 0.50 & 0.825 & 0.17 & 0 \\
\hline 0.75 & 0.35 & 0.65 & 0 \\
\hline 1 & 0.175 & 0.82 & 0 \\
\hline 1.25 & 0.125 & 0.87 & 0 \\
\hline 1.50 & 0.05 & 0.95 & 0 \\
\hline 1.75 & 0.05 & 0.95 & 0 \\
\hline 2 & 0.05 & 0.95 & 0.025 \\
\hline 2.25 & 0.05 & 0.95 & 0.125 \\
\hline 2.50 & 0.05 & 0.95 & 0.225 \\
\hline 2.75 & 0.05 & 0.95 & 0.5 \\
\hline 3 & 0.05 & 0.95 & 0.775 \\
\hline 3.25 & 0.05 & 0.95 & 0.875 \\
\hline 3.50 & 0.025 & 0.97 & 0.95 \\
\hline 3.75 & 0 & 0.97 & 1 \\
\hline 4 & 0 & 0.98 & 1 \\
\hline
\end{tabular}

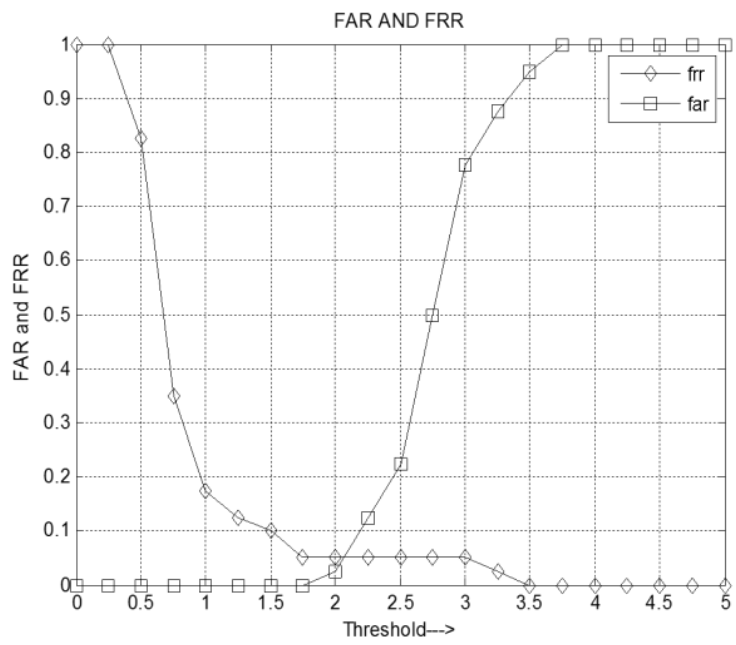

Fig 10: Graph of FRR and FAR with threshold value for L-spacek database

\subsection{Results for JAFFE Database}

The Table 4 shows the variations of FRR, TSR and FAR with threshold. for JAFFE database where out of 10 persons 5 are considered inside the database and 5 persons out of database for testing. FRR and TSR decreases with increase in threshold whereas FAR increases with increase in threshold. As threshold increases, TSR increases from $0 \%$ to $80 \%$. The maximum TSR for JAFFE database is $80 \%$. The Figure 11 . shows the variations of FRR and FAR with threshold. At the threshold value of 5 the FRR is $0 \%$ and FAR is $100 \%$. The TSR for JAFFE database is obtained at a threshold value of 2.15 is $80 \%$. EER obtained at this threshold value is $10 \%$. 
Table 4. FRR, TSR and FAR variations with Threshold for JAFFE database

\begin{tabular}{|c|c|c|c|}
\hline Threshold & FRR & TSR & FAR \\
\hline 0 & 1 & 0 & 0 \\
\hline 0.25 & 1 & 0 & 0 \\
\hline 0.50 & 1 & 0 & 0 \\
\hline 0.75 & 1 & 0 & 0 \\
\hline 1 & 0.8 & 0.2 & 0 \\
\hline 1.25 & 0.4 & 0.6 & 0 \\
\hline 1.50 & 0.4 & 0.6 & 0 \\
\hline 1.75 & 0.4 & 0.6 & 0 \\
\hline 2 & 0.2 & 0.8 & 0 \\
\hline 2.25 & 0 & 0.8 & 0.2 \\
\hline 2.50 & 0 & 0.8 & 0.2 \\
\hline 2.75 & 0 & 0.8 & 0.6 \\
\hline 3 & 0 & 0.8 & 0.6 \\
\hline 3.25 & 0 & 0.8 & 0.8 \\
\hline 3.50 & 0 & 0.8 & 1 \\
\hline 3.75 & 0 & 0.8 & 1 \\
\hline 4 & 0 & 0.8 & 1 \\
\hline
\end{tabular}

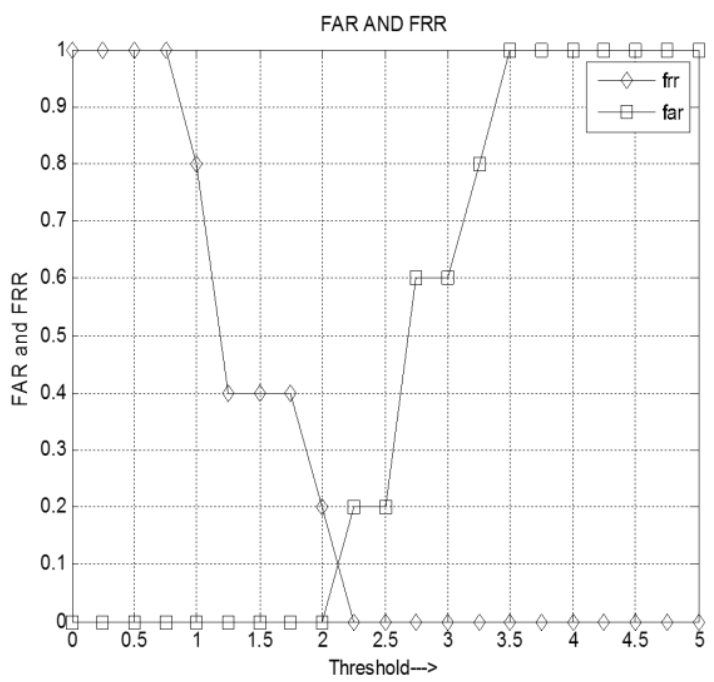

Fig 11: Graph of FRR and FAR with Threshold value for JAFFE database

\subsection{Results for CMU-PIE Database}

The Table 5 shows the variations of FRR, TSR and FAR with threshold for CMU-PIE database where out of 30 persons 15 are considered inside the database and 15 persons out of database for testing. FRR and TSR decreases with increase in threshold whereas FAR increases with increase in threshold. As threshold increases, TSR increases from $0 \%$ to $90 \%$. The maximum TSR for CMU-PIE database is $90 \%$. The Figure 12 shows the variations of FRR and FAR with threshold. At the threshold value of 5 the FRR is $0 \%$ and FAR is $100 \%$. The TSR for CMU-PIE database is obtained at a threshold value of 2.1 is $90 \%$. EER obtained at this threshold value is $10 \%$.
Table 5. FRR, TSR and FAR variations with Threshold for CMU-PIE database

\begin{tabular}{|c|c|c|c|}
\hline Threshold & FRR & TSR & FAR \\
\hline 0 & 1 & 0 & 0 \\
\hline 0.25 & 1 & 0.10 & 0 \\
\hline 0.50 & 0.9 & 0.65 & 0 \\
\hline 0.75 & 0.35 & 0.85 & 0 \\
\hline 1 & 0.15 & 0.90 & 0 \\
\hline 1.25 & 0.1 & 0.90 & 0 \\
\hline 1.50 & 0.1 & 0.90 & 0 \\
\hline 1.75 & 0.1 & 0.90 & 0 \\
\hline 2 & 0.1 & 0.90 & 0 \\
\hline 2.25 & 0.1 & 0.90 & 0.15 \\
\hline 2.50 & 0.1 & 0.90 & 0.25 \\
\hline 2.75 & 0.1 & 0.90 & 0.3 \\
\hline 3 & 0.1 & 0.90 & 0.45 \\
\hline 3.25 & 0.05 & 0.90 & 0.75 \\
\hline 3.50 & 0 & 0.90 & 0.95 \\
\hline 3.75 & 0 & 0.90 & 0.95 \\
\hline 4 & 0 & 0.90 & 1 \\
\hline
\end{tabular}

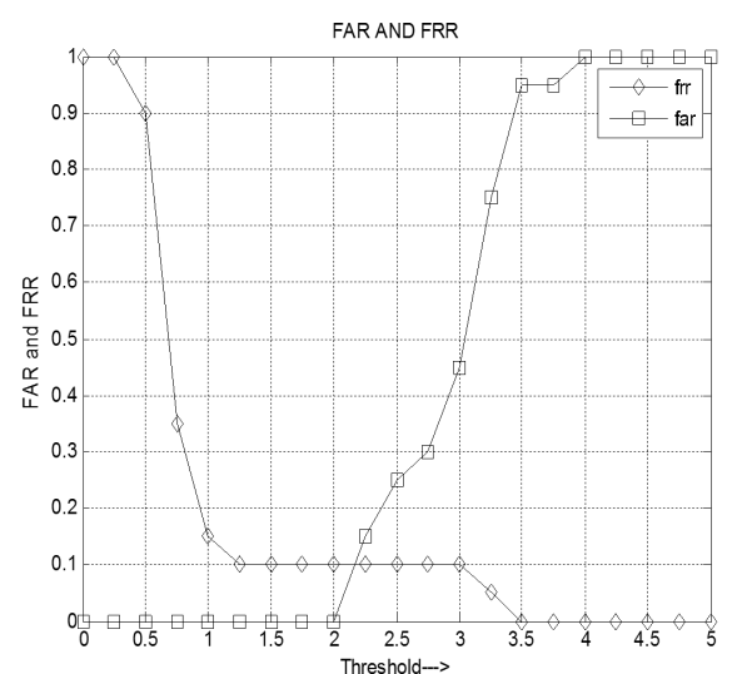

Fig 12: Graph of FRR and FAR with Threshold value for CMU-PIE database

The Recognition Rate of FROCF Algorithm for different database against threshold values is shown in Figure 13.From the graph it is observed that Recognition Rate linearly varies for all considered database within the threshold range of 0 to 2 and Recognition Rate reaches a saturation values of 80,90 ,95 and 97.5 for JAFEE ,CMU-PIE, ORL and L-speck database respectively within a threshold ranging from 2 to 4.The Receiver Operating Characteristics for different features of FROCF Algorithm is shown Figure 14. The ROC consists of plot of FRR versus FAR for ORL.L-Speck, JAFEE and CMU-PIE databases. The FRR values decreases from maximum value to zero and FAR values increases from zero to maximum values as threshold is increasing from zero to maximum value. The ROC Characteristics helps in selecting a 
optimum threshold value which gives good Recognition Rate with less FAR and FRR values.

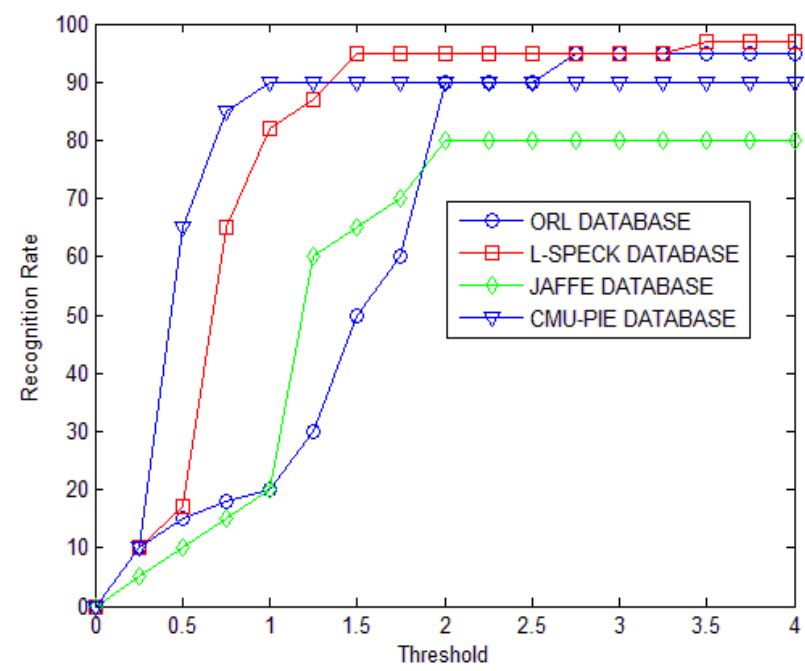

Fig 13: Graph of Recognition Rate of FROCF algorithm for different database

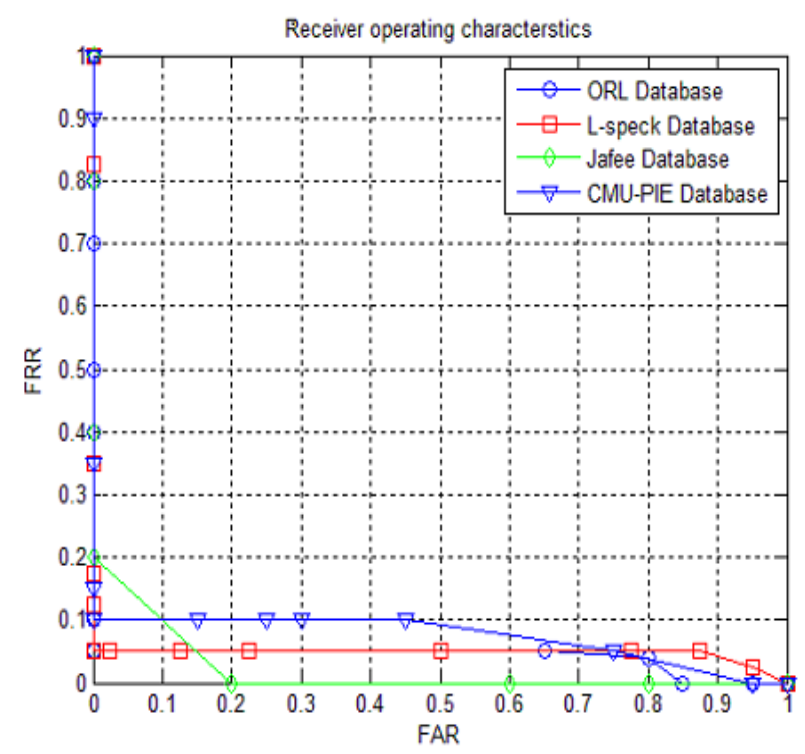

Fig 14: Receiver Operating Characteristics of FROCF Algorithm

\subsection{Comparison of Recognition Rate with Different Algorithms}

The percentage recognition of FROCF algorithm is compared with existing algorithms presented by Gao et al., [9] Li et al., [10] and Huang et al., [11] is given in Table 6 for ORL database. It is observed that the percentage recognition rate is high i.e., 95.3\% compared to low value of existing algorithms [9],[10],[11] for the ORL database. The percentage recognition rate of FRDF is $90.25 \%$ compared to existing algorithm presented in Zhang et al., [12] for CMU-PIE database. The FROCF algorithm performs better for the following reasons: (i)The feature extracted by DTCWT are shift invariant and capture directional variations (ii) The pose and illumination variations are converted into frequency domain using FFT and features are computed by considering absolute values of FFT coefficients. (iii) The features of
DTCWT and FFT are fused using arithmetic addition to obtain final features to identify face images accurately. Table 6 gives Comparison of Recognition Rate of FROCF algorithm with other Existing Algorithms [9],[10],[11] for ORL Database. Table 7. gives the comparison of Recognition Rate of FROCF algorithm in comparison with Zhang et al., [12].

Table 6. Comparison of Recognition Rate of Proposed Algorithm with Various Existing Algorithm for ORL Database

\begin{tabular}{|l|l|c|c|}
\hline $\begin{array}{l}\text { SI } \\
\text { no }\end{array}$ & \multicolumn{1}{|c|}{ Authors } & Techniques & $\begin{array}{c}\text { Recognition } \\
\text { Rate }\end{array}$ \\
\hline 01 & $\begin{array}{l}\text { GAO } \\
\text { Zhirong et } \\
\text { al.,[9] }\end{array}$ & HL_LRC & $93.5 \%$ \\
\hline 02 & $\begin{array}{l}\text { LI YueLong } \\
\text { et al.,[10 ] }\end{array}$ & DSRC-LDA & $88.0 \%$ \\
\hline 03 & $\begin{array}{l}\text { Jun Huang et } \\
\text { al.,[11] }\end{array}$ & MPCA+LDA & $92 \%$ \\
\hline 04 & $\begin{array}{l}\text { Proposed } \\
\text { algorithm }\end{array}$ & DTCWT+FFT & $95.3 \%$ \\
\hline
\end{tabular}

Table 7. Comparison Recognition Rate of FROCF algorithm for CMU-PIE database

\begin{tabular}{|c|c|c|c|}
\hline $\begin{array}{c}\text { SI } \\
\text { no }\end{array}$ & Authors & Techniques & $\begin{array}{c}\text { Recognition } \\
\text { Rate }\end{array}$ \\
\hline 01 & $\begin{array}{c}\text { Jian Zhang et } \\
\text { al.,[12] }\end{array}$ & NOMR & $82.31 \%$ \\
\hline 02 & $\begin{array}{c}\text { Proposed } \\
\text { algorithm }\end{array}$ & DTCWT+FFT & $90.25 \%$ \\
\hline
\end{tabular}

\section{CONCLUSIONS}

Face is a reliable biometric trait used to authenticate a person. In the Proposed FROCF Model shift invariant and directional features of face images are obtained by applying 5-Level DTCWT are combined with pose and illumination variation features of FFT to form Final Features. The Euclidean distance classifier is used to match test image features with database image features and Performance Parameters FAR, FRR and TSR are computed. The Performance of Proposed FROCF shows better performance results compared to Existing Methods .In Future both spatial and frequency domain features are combined for better performance.

\section{ACKNOWLEDGMENTS}

Our thanks to management of Alpha College of Engineering and Jawaharlal Nehru Technological University, Anantapur,for their support in Research.

\section{REFERENCES}

[1] Alaa Eleyan, Hasan Demirel and Hiiseyin O. 2008. Face Recognition using Dual-Tree Wavelet Transform. IEEE 
International Symposium on Signal Processing and Information Technology, pp 7-11.

[2] Zhongxi Sun, Wankou Yang, Changyin Sun and Jifeng, S. 2010. Face Recognition using DTCWT Feature-based 2D PCA .Chinese Conference on Pattern Recognition. pp $1-5$.

[3] Ravi, J. Saleem, S.T. and Raja, K.B. 2012.Face Recognition using DT-CWT and LBP Features. International Conference on Computing, Communication and Applications, pp. 1-6.

[4] Yuehui Sun and Minghui D. 2006. DTCWT Feature based Classification using Orthogonal Neighborhood Preserving Projections for Face Recognition. International Conference on Computational Intelligence and Security. vol.1, pp. 719-724.

[5] Sun Zhongxi, Sun Changyin, ShenJifeng and Yang, W. 2011. Face Recognition using DT-CWT Feature-based 2DIFDA.Chinese Control Conference, pp. 3140-3145.

[6] Ramesha, K. and Raja, K.B. 2011. Performance Evaluation of Face Recognition based on DWT and DTCWT using Multi-Matching Classifiers. International Conference on Computational Intelligence and Communication Networks. pp. 601-605.

[7] Kingsbury, N. G. 2001.Complex Wavelets for Shift Invariant Analysis and Filtering of Signals. Journal of
Applied Computational Harmonic Analysis, vol.10, pp. 234-253.

[8] Muhammad, U. Arif, M. and Ajmal M. 2015. Hyper spectral Face Recognition with Spatiospectral Information Fusion and PLS Regression. IEEE Transactions on Image Processing, vol. 24, no. 3, pp. 1127-1137.

[9] Gao, z. Ding Lixin, Xiong Chengyi and Huang, B. 2014. A Robust Face Recognition Method using Multiple Features Fusion and Linear Regression. Journal of Natural Science, vol.19 no.4, pp. 323-327.

[10] yuelong, Li. Meng Li, Feng Jufu and Jigang,2014. Downsampling sparse representation and discriminant information aided occluded face recognition. International Journal of Science China Information Sciences, vol. 57 ,no.03, pp. 1-8.

[11] Jun Huang, Kehua Su, Jamal El-Den, Tao Hu, and Junlong, Li. 2014. An MPCA/LDA Based Dimensionality Reduction Algorithm for Face Recognition. International Journal on Mathematical Problems in Engineering, vol.14, pp. 1-12.

[12] Jian Zhang ,JianYang, JianjunQian and Jiawei,Xu,2014. Nearest orthogonal matrix representation for face recognition. International Journal on Neurocomputing, vol.151, pp. 471-480 